\title{
Factores de riesgo asociados con Apgar bajo al nacer en el Hospital Hipólito Unanue
}

\author{
Risk Factors Associated With Low Apgar Scores at Birth at the Hipólito Unanue Hospital \\ Fatores de risco associados ao Apgar baixo ao nascer no Hospital Hipólito Unanue
}
Alexander Chambilla-Coila ${ }^{1 a}$
(D) https://orcid.org/0000-0002-7313-3117
Manuel Ticona-Rendón ${ }^{2 b}$
(D) https://orcid.org/0000-0002-4819-1251
Diana Huanco-Apaza ${ }^{2 c}$
https://orcid.org/ 0000-0001-9687-8384

\section{Resumen}

Objetivo: Determinar los factores de riesgo asociados con Apgar bajo al nacer en el Hospital Hipólito Unanue de Tacna. Material y Método: Estudio analítico de casos y controles. Se incluyó a todos los recién nacidos vivos cuyos partos fueron atendidos en el Hospital Hipólito Unanue de Tacna durante los años 2008 a 2017. Los casos fueron recién nacidos con Apgar al minuto menor de 7 y controles con Apgar de 7 a 10. Para el análisis estadístico se utilizó comparación de frecuencias absolutas y relativas, Odds Ratio con intervalo de confianza y valor de "p". La información se obtuvo de la base de datos del Sistema Informático Perinatal. Resultados: La incidencia de Apgar bajo al nacer fue de 4,4 \%. Los factores de riesgo socioeconómicos fueron: edad materna 35 años o más $(O R=1,4)$, instrucción primaria $(O R=1,2)$, estado civil soltera $(O R=1,3)$. Los factores de riesgo obstétricos: gran mutiparidad $(O R=1,4)$, morbilidad materna $(O R=1,3)$, polihidramnios $(O R=11,9)$, diabetes mellitus $(O R=4,7)$, presentación fetal anómala $(O R=3,4)$, hemorragia del tercer trimestre $(O R=3,3)$, ruptura prematura de membranas $(O R=1,9)$, oligoamnios $(O R=1,7)$ y enfermedad hipertensiva del embarazo $(O R=1,5)$. Los factores de riesgo del parto: cesárea $(O R=1,3)$, parto precipitado $(O R=6,6)$, líquido amniótico verde oscuro $(O R=2,5)$ y líquido amniótico verde claro $(O R=1,8)$. Los factores de riesgo perinatales: sexo masculino $(O R=1,4)$, bajo peso al nacer $(O R=10,9)$, peso insuficiente $(O R=1,7)$, alto peso $(O R=1,2)$, prematuridad $(O R=9,0)$, malformación congénita $(O R=7,3)$, retardo de crecimiento intrauterino $(O R=6,1)$ y gemelaridad $(O R=2,3)$. Conclusiones: Los factores de riesgo que se asocian con Apgar bajo al nacer fueron principalmente factores perinatales y obstétricos.

Palabras clave: asfixia neonatal, factores de riesgo, puntaje de Apgar [Fuente: DeCS]

\section{Abstract}

Objective: To determine the risk factors associated with low Apgar at birth at Hipólito Unanue Hospital in Tacna, Perú. Material and Method: Analytical case-control study-in which all live newborns whose deliveries were attended at the Hipólito Unanue de Tacna during the years 2008 to 2017 were included. The cases were newborns with Apgar score of less than 7 and the controls with Apgar scores of 7 to 10. For the statistical analysis, comparison of absolute and relative frequencies, Odds Ratio with confidence interval and "p" value was used. The information was obtained from the Perinatal Information System database. Results: The incidence of low Apgar at birth was $4.4 \%$. The socioeconomic risk factors were: maternal age 35 years or more $(\mathrm{OR}=1.4)$, primary education $(O R=1.2)$, single marital status $(O R=1.3)$. Obstetric risk factors: great mutiparity $(O R=1.4)$, maternal

\footnotetext{
${ }^{1}$ Investigador independiente

${ }^{2}$ Facultad de Ciencias de la Salud, Universidad Nacional Jorge Basadre Grohmann. Tacna, Perú

${ }^{a}$ Médico Cirujano

${ }^{\mathrm{b}}$ Doctor en Medicina y Salud Pública

${ }^{\circ}$ Doctora en Salud Pública
} 
morbidity $(O R=1.3)$, polyhydramnios $(O R=11.9)$, diabetes mellitus $(O R=4.7)$, abnormal fetal presentation $(O R==3.4)$, third trimester hemorrhage $(O R=3.3)$, premature rupture of membranes $(O R=1.9)$, oligoamnios $(O R=1.7)$ and hypertensive disease of pregnancy $(O R=1.5)$. Risk factors for delivery: cesarean section $(O R=1.3)$, precipitated delivery $(O R=6.6)$, dark green amniotic fluid $(O R=$ $2.5)$, and light green amniotic fluid $(O R=1.8)$. Perinatal risk factors: male sex $(O R=1.4)$, low birth weight $(O R=10.9)$, underweight $(O R=1.7)$, high weight $(O R=1.2)$, prematurity $(O R=9.0)$, congenital malformation $(\mathrm{OR}=7.3)$, intrauterine growth retardation $(\mathrm{OR}=6.1)$ and twinning $(\mathrm{OR}=$ 2.3). Conclusions: The risk factors associated with low Apgar at birth were mainly perinatal and obstetric factors.

Keywords: asphyxia neonatorum, risk factors, apgar score [Source: MeSH]

\section{Resumo}

Objetivo: Determinar os fatores de risco associados ao baixo Apgar ao nascer no Hospital Hipólito Unanue de Tacna. Material e Método: Estudo analítico de casos e controles. Foram incluídos todos os recém-nascidos vivos cujos partos foram atendidos no Hospital Hipólito Unanue de Tacna nos anos de 2008 a 2017. Os casos eram recém-nascidos com índice de Apgar menor que 7 e os controles com índice de Apgar de 7 a 10. Para a análise estatística, foi utilizada a comparação das frequências absolutas e relativas, Odds Ratio com intervalo de confiança e valor "p". As informações foram obtidas no banco de dados do Sistema de Informação Perinatal. Resultados: A incidência de Apgar baixo ao nascimento foi de $4,4 \%$. Os fatores de risco socioeconômicos foram: idade materna de 35 anos ou mais $(O R=1,4)$, ensino fundamental $(O R=1,2)$, estado civil solteiro $(O R=1,3)$. Fatores de risco obstétricos: grande mutiparidade $(O R=1,4)$, morbidade materna $(O R=1,3)$, poliidrâmnio $(O R=11,9)$, diabetes mellitus $(O R=4,7)$, apresentação fetal anormal $(O R==3,4)$, hemorragia de terceiro trimestre $(O R=3,3)$, ruptura prematura de membranas $(O R=1,9)$, oligoamnios $(O R=1,7)$ e doença hipertensiva da gravidez $(O R=1,5)$. Fatores de risco para parto: cesariana $(O R=1,3)$, parto precipitado $(O R=6,6)$, líquido amniótico verde escuro $(O R=2,5)$ e líquido amniótico verde claro $(O R=1,8)$. Fatores de risco perinatais: sexo masculino $(O R=1,4)$, baixo peso ao nascer $(O R=10,9)$, baixo peso $(O R=1,7)$, alto peso $(O R=1,2)$, prematuridade $(O R=9,0)$, malformação congênita $(O R=7,3)$, retardo de crescimento intrauterino $(O R=6,1)$ e geminação $(O R=$ $2,3)$. Conclusões: Os fatores de risco associados ao baixo Apgar ao nascer foram principalmente fatores perinatais e obstétricos.

Palavras-chave: asfixia neonatal, fatores de risco, índice de Apgar [Fonte: DeCS]

\section{Introducción}

La mayoría de los embarazos y partos tienen una evolución fisiológica lo cual constituye un evento normal. ${ }^{1}$ El periodo crucial de la vida humana se traduce en las primeras 24 horas después del nacimiento, durante el cual el neonato establece adaptaciones cardiopulmonares necesarias para asegurar un intercambio gaseoso adecuado, además cambios renales, hepáticos y metabólicos indispensables para responder a las exigencias de la vida extrauterina; por lo antes mencionado, la morbilidad y mortalidad neonatal en este periodo son elevadas. ${ }^{2}$
Desde 1952, la doctora Virginia Apgar elaboró un sistema de evaluación conocido como el test de Apgar que permite establecer el estado general del recién nacido en el momento del nacimiento y toma en cuenta 5 parámetros: frecuencia cardiaca, esfuerzo respiratorio, coloración de la piel, tono muscular y respuesta refleja. ${ }^{3}$ Este puntaje, universalmente usado, es una medida objetiva e indicador de bienestar del niño al nacimiento; desarrollado para identificar rápidamente los recién nacidos que necesitan reanimación. Si un recién nacido presenta puntaje menor de 7 al minuto y persiste a los 5 minutos, se considera un evento que, si no se proporciona una adecuada y 
oportuna reanimación, pone en riesgo de muerte o secuelas neurológicas. ${ }^{4}$

La depresión respiratoria al nacer es una de las causas más frecuentes de ingreso a unidades de cuidados intensivos y una de las principales urgencias al momento de nacimiento que obliga a tomar medidas urgentes y enérgicas para disminuir la morbimortalidad neonatal. ${ }^{5}$ Constituye un problema que afecta a 2-5\% de todos los nacidos vivos ${ }^{2,6}$ y ocupa un lugar muy importante como una de las principales causas de mortalidad perinatal. ${ }^{5}$

La gran mayoría de las causas de hipoxia perinatal son de origen intrauterino, aproximadamente el $5 \%$ ocurre antes del inicio del trabajo de parto, $85 \%$ durante el parto y el periodo expulsivo y el $10 \%$ restante durante el periodo neonatal. Son muchos los factores que se han implicado en la depresión al nacer entre los que se encuentran las afecciones propias del embarazo como la prematuridad, rotura prematura de membranas, enfermedad hipertensiva del embarazo, diabetes gestacional, embarazo prolongado, embarazo múltiple entre otras. Otros factores son los relacionados a afecciones de los anexos ovulares y fetales como los infartos placentarios, placenta previa, compresión del cordón umbilical, alteraciones en la cantidad del líquido amniótico, bajo peso, sufrimiento fetal y malformaciones congénitas. También existen factores como el estado socioeconómico de la madre, edades extremas, hábitos de fumar y nutrición materna que pueden influir en la evaluación de Apgar. ${ }^{1,5}$

El logro de un recién nacido como resultado de un parto con el menor riesgo posible para el desarrollo futuro siempre ha sido el objetivo principal de los profesionales de la salud, para ello es necesario asegurar un trabajo de parto con calidad y un nacimiento seguro para disminuir considerablemente la tasa de mortalidad perinatal y lograr un aumento en la calidad de vida. ${ }^{7}$
El conocimiento de los factores de riesgo del Apgar bajo debería contribuir a mejorar el cuidado antenatal, basado en la mejor identificación del alto riesgo de las mujeres embarazadas; ${ }^{8}$ por ello, el propósito de este estudio fue determinar los factores de riesgo asociados con Apgar bajo al nacer en el Hospital Hipólito Unanue de Tacna.

Estudio analítico, de casos y controles. La población de estudio estuvo constituida por todos los recién nacidos vivos con peso al nacer de 500 gramos a más, cuyo nacimiento fue atendido en el Hospital Hipólito Unanue de Tacna, durante los años 2008 a 2017. Este hospital es el único del Ministerio de Salud de la región y atiende a la población de bajos y medianos recursos económicos y el $99 \%$ de la población materno perinatal atendida cuenta con Seguro Integral de Salud (SIS). Aproximadamente el $70 \%$ de los partos de toda la región se atienden en este hospital.

Los casos estuvieron conformados por todos los recién nacidos vivos con Apgar al minuto de vida menor de 7 , y los controles por todos los recién nacidos vivos con Apgar al minuto de 7 a 10. La información se obtuvo de la base de datos del Sistema Informático Perinatal (SIP2000).

Para el análisis estadístico bivariado, se comparó frecuencias absolutas y relativas entre ambos grupos, y se utilizó el Odds Ratio (OR) con intervalo de confianza (IC) al $95 \%$ de confiabilidad y el valor de " $p$ ", considerando factor de riesgo significativo cuando el IC fue mayor de 1 y el $p$ menor de 0,05.

Para el procesamiento y análisis de datos se utilizó el software del SIP2000 y Epiinfo.

\section{Resultados}

En el Hospital Hipólito Unanue de Tacna durante los años 2008 a 2017 nacieron 34618 productos vivos, de los cuales 1513 presentaron al minuto de nacer Apgar menor de 7 que representa $4,4 \%$, oscilando de $5,5 \%$ en 
el año 2009 a $4 \%$ en el año 2017 , con una tendencia descendente. El $70 \%$ presentó Apgar de 4 a 6 y $30 \%$ Apgar de 0 a 3. Tabla y Figura 1.

Los factores de riesgo sociodemográficos asociados significativamente a Apgar bajo al nacer (Tabla 2) fueron: edad materna de 35 años a más (OR $=1,4)$, grado de instrucción primaria $(O R=1,2)$ y estado civil soltera $(O R=1,3)$.

Los factores de riesgo obstétricos asociados significativamente a Apgar bajo al nacer (Tabla 3) fueron: gran multiparidad (OR = $1,4)$, morbilidad materna $(O R=1,3)$, patologías maternas como polihidramnios $(\mathrm{OR}=11,9)$, diabetes mellitus $\mathrm{OR}=4,7$ ), presentación fetal anómala $(O R=3,4)$, hemorragia del tercer trimestre $(O R=3,3)$, ruptura prematura de membranas $(\mathrm{OR}=1,9)$, oligohidramnios $(\mathrm{OR}=$ $1,7)$ y enfermedad hipertensiva del embarazo $(\mathrm{OR}=1,5)$.

Durante el parto, los factores que se asociaron a Apgar bajo (Tabla 4) fueron: terminación por cesárea $(O R=1,3)$, líquido amniótico verde oscuro $(\mathrm{OR}=2,5)$, líquido amniótico verde claro $(\mathrm{OR}=1,8)$ y parto precipitado $(\mathrm{OR}=6,6)$.

Los factores de riesgo perinatales asociados significativamente (Tabla 5) fueron: sexo masculino $(\mathrm{OR}=1,4)$, bajo peso al nacer $(O R=10,9)$, peso insuficiente $(O R=1,7)$, alto peso $(\mathrm{OR}=1,2)$, pretérmino $(\mathrm{OR}=9,0)$, malformación congénita $(O R=7,3)$, retardo de crecimiento intrauterino $(O R=6,1) \mathrm{y}$ gemelaridad $(\mathrm{OR}=2,3)$.

\section{Discusión}

En el Hospital Hipólito Unanue de Tacna, durante los últimos diez años, el 4,4 \% de recién nacidos vivos presentaron Apgar al minuto menor de 7 , siendo los principales factores de riesgo perinatales asociados a este evento: bajo peso al nacer, prematuridad, malformaciones congénitas y retardo de crecimiento intrauterino. Seguido de factores de riesgo obstétricos como polihidramnios, diabetes mellitus, enfermedad hipertensiva del embarazo, hemorragia del tercer trimestre, ruptura prematura de membranas y presentación fetal anómala. Durante el parto se asociaron con Apgar bajo: la cesárea, líquido amniótico verde y parto precipitado. Adicional a ello, se asociaron factores de riesgo socioeconómicos negativos como edad materna avanzada, bajo grado de instrucción y estado civil soltera.

A nivel internacional, la frecuencia de Apgar bajo al minuto de nacimiento varía. Así, León ${ }^{7}$ en el hospital Materno de Referencia Nacional de Asmara en Eritrea durante el 2006 a 2007 encontró que los recién nacidos con Apgar bajo representó el 1,54\%. Montero ${ }^{9}$ reportó que el 3,1\% en el Hospital Gineco Obstétrico de Guanabacoa en la Habana Cuba en el 2009. Amaya $^{10}$ reportó el $1,4 \%$ en el Hospital Nacional de Zacamil en El Salvador. Tales cifras fueron menores a lo encontrado en este estudio. Contrariamente, otros autores encontraron cifras superiores. Por un lado, Rodríguez ${ }^{11}$ reportó un $11 \%$ en el Hospital Gineco Obstétrico Eusebio Hernandez Pérez en la Habana Cuba entre el 2004 a 2012. Por otro lado, Omokhodion ${ }^{12}$ reportó un $20 \%$ en cuatro hospitales en Ibadan Nigeria. Por lo expuesto, se deduce que la frecuencia de Apgar bajo se relaciona a las condiciones de vida, nivel de desarrollo y a los recursos humanos y tecnológicos de cada centro hospitalario.

Al analizar los factores de riesgo sociodemográficos del Apgar bajo al nacer encontramos asociación con edad materna avanzada, semejante hallazgo encontró Flores (13) en el Hospital César Garayar García lquitos Perú en el año $2016(\mathrm{OR}=1,9)$. Las mujeres de más de 35 años son más vulnerables a enfermedades crónicas y cromosomopatías; ${ }^{5}$ además, existe un deterioro de la función miometrial, lo cual trae consigo que se vea comprometida la implantación, transporte de sustancias al feto que compromete su nutrición y oxigenación lo que influye en la depresión al nacer. ${ }^{2}$ 
Las gestantes con estado civil soltera tienen mayor probabilidad de que sus recién nacidos presenten Apgar bajo al nacer, debido a que no cuentan con un apoyo emocional de su pareja y familia para llevar un adecuado control pre natal, además de presentar mayores complicaciones durante su embarazo y parto. Tal como lo reporta Carpio, ${ }^{14}$ quien encontró que el estado civil soltera fue de $18,1 \%$ en recién nacidos con Apgar bajo en el hospital Regional Hermilio Valdizán Medrano de Huánuco en el 2014. Existe poca información sobre las determinantes socioeconómicas, ambientales $\mathrm{u}$ ocupacionales de los bajos puntajes de Apgar. Un ejemplo de determinante socioeconómico es el estudio de Omokhodion ${ }^{12}$ en Nigeria, quien encontró al bajo grado de instrucción como un factor de riesgo al igual que en nuestro estudio.

La gran multiparidad constituye un factor de riesgo obstétrico asociado a Apgar bajo al nacer. Semejante resultado fue encontrado por Peralta ${ }^{15}$ en el Hospital Regional Manuel Núñez Butrón de Puno en el año 2017 en madres con 5 gestaciones a más $(\mathrm{OR}=1,2)$.

Cuando analizamos las patologías maternas encontramos entidades que pueden comprometer el flujo útero placentario como la enfermedad hipertensiva del embarazo o aquellas que pueden desencadenar el parto antes del término de la gestación y dar como resultado un producto con una pobre adaptación al medio extrauterino y puntaje de Apgar bajo al nacer. La adaptación neonatal depende de factores como la edad gestacional al nacer, el peso fetal, las enfermedades médicas maternas e inducidas por el embarazo. ${ }^{1}$

Carpio ${ }^{14}$ encontró asociación con Apgar bajo al nacer a la enfermedad hipertensiva del embarazo, placenta previa y presentación podálica $(p<0,05)$ en Huánuco. Quiroz ${ }^{16}$ encontró asociación con diabetes mellitus (OR $=4,4$ ) en el Hospital Regional de Pucallpa en el 2017. Peralta, ${ }^{15}$ en Puno y Arana ${ }^{17}$ en Trujillo encontraron asociación con presentación anómala. Asimismo, Flores ${ }^{13}$ reportó asociación con enfermedad hipertensiva del embarazo $(\mathrm{OR}=1,5)$ en lquitos. Salvo ${ }^{4}$ informó sobre asociación con DPP, RPM, patología de cordón $y$ enfermedad hipertensiva del embarazo $(p<$ 0,01 ) en Chile. Suka ${ }^{8}$ encontró asociación con preeclampsia en el hospital de Japón en los años 1993 a 1995. Kovavisarach ${ }^{18}$ registró asociación con presentación podálica en 1995 y 1996 en el hospital Rajavithi en Tailandia. Omokhodion $^{12}$ registró asociación con hipertensión en el embarazo, RPM y presentación de nalgas en Nigeria.

Existe relación entre el tiempo prolongado de ruptura de membranas y la posibilidad de sepsis en el recién nacido, con la consecuente aparición de Apgar bajo; además, la rotura de membranas puede llevar directamente a la presencia de Apgar bajo al nacer cuando ocurre un accidente como la procidencia del cordón umbilical, aunque este evento es poco frecuente. ${ }^{19}$

La hipertensión compromete el flujo uteroplacentario, dando como resultado un producto con una pobre adaptación al medio extrauterino y puntaje de Apgar bajo al nacer. ${ }^{5}$

Durante el parto, diversos autores encontraron a la cesárea como factor de riesgo asociado al Apgar bajo, tales como Arana $^{17}$ en Trujillo (OR $=3,5)$, Bandera $^{2}$ en el hospital Materno "Mariana Grajales" en Santiago de Cuba en el 2006 a 2007 (OR = 3,2), Salvo en Chile, Amaya $^{10}$ en El Salvador y Omokhodion ${ }^{12}$ en Nigeria. La vía de parto por cesárea presenta mayor riesgo de recién nacidos con Apgar bajo al nacer debido a que la indicación de cesárea se debe principalmente a la presencia de alguna patología materna o perinatal.

La presencia del líquido amniótico meconial constituye un factor de riesgo para Apgar bajo al nacer; semejantes resultados fueron encontrados por Arana ${ }^{17}$ en Trujillo (OR = $4,1)$, Bandera ${ }^{2}$ en Cuba (OR $\left.=3,5\right)$, Salvo ${ }^{4}$ en Chile y Kovavisarach ${ }^{18}$ en Tailandia. Esto porque frecuentemente se asocia al sufrimiento 
fetal agudo o crónico con su repercusión sobre elApgar al nacer. ${ }^{19}$

Al analizar la duración del parto vaginal, Montero ${ }^{9}$ encontró que el parto precipitado (OR $=3,8$ ) constituyó un factor asociado a Apgar bajo al nacer al igual que en este estudio. La duración del parto precipitado presenta mayor riesgo de recién nacidos con Apgar bajo al nacer debido a que conlleva a la hipoxia fetal por deficiencia de oxigenación.

Dentro de los factores perinatales, se encuentra que el recién nacido de sexo masculino es un factor de riesgo asociado a Apgar bajo. Montero encontró que el $75,5 \%$ de recién nacidos con Apgar bajo al nacer fueron de sexo masculino. Álvarez $z^{5}$ encontró que la mayoría de los recién nacidos deprimidos fueron de sexo masculino (57,6 \%), debido a que los neonatos masculinos presentan mayor vulnerabilidad a los trastornos respiratorios perinatales.

El peso del recién nacido como bajo, insuficiente y alto peso al nacer son un factor de riesgo para Apgar bajo. El bajo peso fue encontrado por Arana ${ }^{17}$ en Trujillo $(\mathrm{OR}=3,4)$, Flores $^{13}$ en lquitos $(\mathrm{OR}=11,4)$, León ${ }^{7}$ en Eritrea y Kovavisarach ${ }^{18}$ en Tailandia. Este último también encontró asociación con el alto peso al nacer. Los productos con bajo peso soportan peor la adaptación a la vida extrauterina. ${ }^{1}$

La prematuridad es un factor de riesgo importante para Apgar bajo, este hallazgo fue encontrado por Arana $^{17}$ en Trujillo $(\mathrm{OR}=4,26)$, Flores $1^{3}$ en Iquitos $(\mathrm{OR}=3,4)$, Salvo ${ }^{4}$ en Chile $(p<0,001)$ y Kovavisarach ${ }^{18}$ en Tailandia. Los recién nacidos pretérmino presenta mayor riesgo de Apgar bajo al nacer debido a que no existe aún un desarrollo adecuado del recién nacido para la vida extrauterina, ni una maduración orgánica. La prematuridad y su problema fundamental, la enfermedad de membrana hialina, unido al escaso desarrollo de la musculatura que interviene en la respiración, dificultan el inicio y mantenimiento de la respiración al nacer; en consecuencia, estos factores propician la puntuación baja del Apgar, además de su inmadurez neurológica, pueden afectar el tono muscular y la respuesta refleja. ${ }^{11,19}$

Los neonatos con bajo peso al nacer y prematuros soportan menos la adaptación a la vida extrauterina y son más propensos a la asfixia, ya que su sistema nervioso es frágil y sensible, e incluso el mismo trabajo de parto puede conllevarlos a desarrollar hipoxia neonatal. ${ }^{5}$

Al analizar las patologías asociadas del recién nacido, se encontró que la malformación congénita, retardo de crecimiento intrauterino y gemelaridad son factores de riesgo. Bandera ${ }^{2}$ encontró asociación con retado de crecimiento intrauterino $(\mathrm{OR}=11,2)$ en Cuba, Quiroz ${ }^{16}$ en Pucallpa con gemelaridad (OR $=2,52)$ y Salvo ${ }^{4}$ en Santiago de Chile con RCIU y malformaciones congénitas ( $p<0,001)$.

Se concluye que la incidencia de recién nacidos con Apgar bajo al nacer en el Hospital Hipólito Unanue de Tacna entre los años 2008 al 2017 fue de 4,4\%, con una tendencia descendente durante los diez años. Los factores de riesgo perinatales fueron los más importantes para el desarrollo de Apgar bajo al minuto, tales como bajo, insuficiente y alto peso al nacer, prematuridad, malformaciones congénitas, retardo de crecimiento intrauterino y gemelaridad. Las patologías maternas que se asociaron fueron alteraciones del líquido amniótico, diabetes mellitus, hemorragia del tercer trimestre, ruptura prematura de membranas y enfermedad hipertensiva del embarazo. En menor proporción se asociaron factores de riesgo sociodemográficos como edad materna avanzada, bajo grado de instrucción y estado civil soltera.

Se recomienda que los resultados de este estudio sean tomados en cuenta como base para desarrollar estrategias preventivas que favorezcan al recién nacido a corto y mediano plazo. Que las complicaciones durante el embarazo y parto deben ser monitoreado adecuadamente por profesional médico para evitar un recién nacido con Apgar bajo al nacer. Dada la importancia de las asociaciones definidas en la presente investigación, se recomienda la realización de estudios multicéntricos con mayor muestra 
poblacional, además de estudios anuales con el fin de que se pueda conocer el comportamiento de la tendencia de los factores de riesgo estudiados con mayor precisión en el tiempo.

\section{Referencias}

1. Laffita A. Factores que influyen en el Apgar bajo al nacer, en el Hospital América Arias de La Habana Cuba, 2000. Rev Chil Obstet Ginecol 2005; 70(6): 359-363. http://dx.doi.org/10.4067/S071775262005000600002

2. Bandera N, Goire M, Cardona O. Factores epidemiológicos y Apgar bajo al nacer. Revista Cubana de Obstetricia y Ginecología 2011; 37(3): 320-329. ISSN0138-600X.

3. Llanos A, Riquelme R. Asfixia perinatal y su repercusión en la asfixia sistémica. En: Meneghello J, Fanta E, Paris E, Puga T. Pediatría Meneghello. 5ta. ed. Tomo I. Santiago de Chile: Ed. Médica Panamericana; 2002. p. 486-87.

4. Salvo H, Flores J, Alarcón J, Nachar R, Paredes A. Factores de riesgo de test de Apgar bajo en recién nacidos. Rev Chil Pediatr 2007; 78(3): 253-60. http://dx.doi.org/10.4067/S037041062007000300003

5. Álvarez C, Ruíz M, Hernández J, Valdés M. Factores influyentes en la depresión neonatal en Santa Cruz del Norte. Rev Ciencias Médicas (La Habana) 2014; 20 ( 3 ): $\quad 367-77$. http://revcmhabana.sld.cu/index.php/rcm h/article/view/662

6. Gonzales H. Asfixia del recién nacido. En: Pérez A, Donoso E. Obstetricia. 3ra. ed. Santiago de Chile: Ed. Mediterráneo; 2001. p. 927-36.

7. León A, Ysidrón E. Factores relacionados con el Apgar bajo al nacer. Rev Cub Obstet Ginecol. 2010; 36(1): 25-35. http://scielo.sld.cu/scielo.php?script=sci a $r \mathrm{t} \mathrm{te} \times \mathrm{t} \& \mathrm{p}$ i d $=\mathrm{S} 0138$ $600 \times 2010000100005 \&$ Ing $=$ es.
8. Suka $M$, Sugimori $H$, Nakamura $M$, Haginiwa K, Yoshida K. Risk factors of low Apgar score in Japanese full-term deliveries: A case-control study. J Epidemiol. 2002; 12(4):320-23. doi: 10.2188/jea.12.320. PMID: 12395872.

9. Montero Y, Balleste I, Vizcano M, Izquierdo M. Depresión neonatal a término relacionada con factores maternos, Hospital Ginecoobstétrico de Guanabacoa, 2009. Rev Cubana Invest Bioméd. 2011; 30 (4): 471-477. http://scielo.sld.cu/scielo.php?script=sci a r t text \& p i d = S 0864 03002011000400005\&lng=es.

10. Amaya A, Arévalo J, López E. Factores prenatales e intraparto relacionados con puntaje de Apgar bajo en el Hospital Nacional de Zacamil. Crea Cienc 2005; 2 ( 3 ) : $\quad 38-43$. https://www.imbiomed.com.mx/ejemplar. php?id=5162

11. Rodríguez Y, Castillo A, López E, Montes E, Arenas C, Rodríguez N. Morbilidad y mortalidad en recién nacidos con depresión. Rev Cubana Ginecol Obstet. $2014 ; 40(4)$ ): $358-67$. http://scielo.sld.cu/scielo.php?script=sci

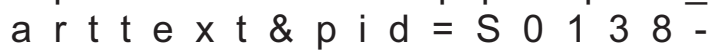
$600 \times 2014000400002 \&$ Ing $=$ es.

12. Omokhodion FO, Roberts OA, Onadeko MO, Beach JR, Cherry N, Burstyn I. Social, obstetric and environmental determinants of low Apgar score among infants born in four selected hospitals in Ibadan, Nigeria. J Obstet Gynaecol. 2018; 38 ( 4 ): $454-460$. d o i : 10.1080/01443615.2017.1367764. Epub 2018 Feb 1. PMID: 29390911.

13. Flores $\mathrm{P}$. Factores de riesgo asociados a Apgar bajo en recién nacidos atendidos en el Hospital Iquitos Cesar Garayar García; Enero-Mayo 2016. 59 p. [Tesis para optar el título profesional de Obstetra]. Iquitos: Universidad Científica del Perú; 2017. http://repositorio.ucp.edu.pe/bitstream/ha ndle/UCP/188/FLORES-1-Trabajo- 
Factores. pdf?sequence $=1$ \&isAllowed $=\mathrm{y}$

14. Carpio E. Factores relacionados con Apgar bajo al nacer en neonatos atendidos del hospital Regional Hermilio Valdizán Medrano Huánuco, 2014. [Tesis para optar el título profesional de Enfermera]. Huánuco: Universidad de $\mathrm{H}$ u á n u c o ; $2016.96 \%$. http://repositorio.udh.edu.pe/123456789/ 97

15. Peralta V. Factores de riesgo asociados a puntaje de Apgar bajo en el servicio de neonatología del Hospital Regional Manuel Núñez Butrón de enero a diciembre del año 2017. [Tesis para optar el título profesional de Médico Cirujano]. Puno: Universidad Nacional del Altiplano; 2018 . $109 \quad \mathrm{p}$. http://repositorio.unap.edu.pe/bitstream/ handle/UNAP/6407/Peralta_Ticona_Vale ntin_Ronald.pdf?sequence $=1$ \&isAllowed $=y$

16. Quiroz F. Diabetes gestacional como factor de riesgo para Apgar bajo al nacer en el Hospital Regional de Pucallpa. [Tesis para optar el título profesional de Médico Cirujano]. Trujillo Perú: Universidad Privada Antenor Orrego;
$2017 . \quad 37 \quad p$. http://repositorio.upao.edu.pe/handle/up aorep/2737

17. Arana L. Factores de riesgo asociados a puntaje Apgar bajo al nacer en neonatos del hospital Belén de Trujillo periodo Enero 2009 - Diciembre 2013. [Tesis para optar el título profesional de Médico Cirujano]. Trujillo Perú: Universidad Privada Antenor Orrego; 2014. 51 p. http://repositorio.upao.edu.pe/handle/up aorep/452

18. Kovavisarach $\mathrm{E}$, Juntasom $\mathrm{C}$. Risk factors of delivery of low Apgar score newborn below 7 at 1 minute: a case-control study. J Med Assoc Thai. 1999; 82(7): 660-65. P M I D : $\quad 10511767$. https://pubmed.ncbi.nlm.nih.gov/1051176 7/

Laffita A, Ariosa J, Cutié J. Apgar bajo al nacer y eventos del periparto. Rev Cubana Obstet Ginecol 2004; 30 (1). Disponible en: Disponible en: http://scielo.sld.cu/scielo.php?script=sci_ a $r$ t $\mathrm{e} \times \mathrm{t} \& \mathrm{p}$ i d = S 0138 $600 \times 2004000100002 \&$ Ing $=e s$.

\section{Correspondencia:}

manuelticonar@yahoo.es
Fecha de recepción: 10 de julio de 2020

Fecha de aceptación: 05 de diciembre de 2020 


\section{Tabla 1}

Frecuencia de Apgar Bajo al minuto. Hospital Hipólito Unanue de Tacna

\begin{tabular}{cccc}
\hline Año & $\begin{array}{c}\text { Nacidos } \\
\text { Vivos }\end{array}$ & $\begin{array}{c}\text { RN con Apgar al } \\
1^{\prime}<7\end{array}$ & $\begin{array}{c}\text { Frecuencia } \\
\text { X 100 nv }\end{array}$ \\
\hline 2008 & 3522 & 155 & 4,4 \\
2009 & 3655 & 202 & 5,5 \\
2010 & 3511 & 153 & 4,4 \\
2011 & 3374 & 111 & 3,3 \\
2012 & 3494 & 160 & 4,6 \\
2013 & 3512 & 168 & 4,8 \\
2014 & 3275 & 102 & 3,1 \\
2015 & 3479 & 192 & 5,5 \\
2016 & 3392 & 131 & 3,9 \\
2017 & 3490 & 139 & 4,0 \\
\hline $2008-2017$ & 34704 & 1513 & 4,4 \\
\hline
\end{tabular}

\section{Figura 1}

Frecuencia de Apgar bajo al minuto. Hospital Hipólito Unanue de Tacna

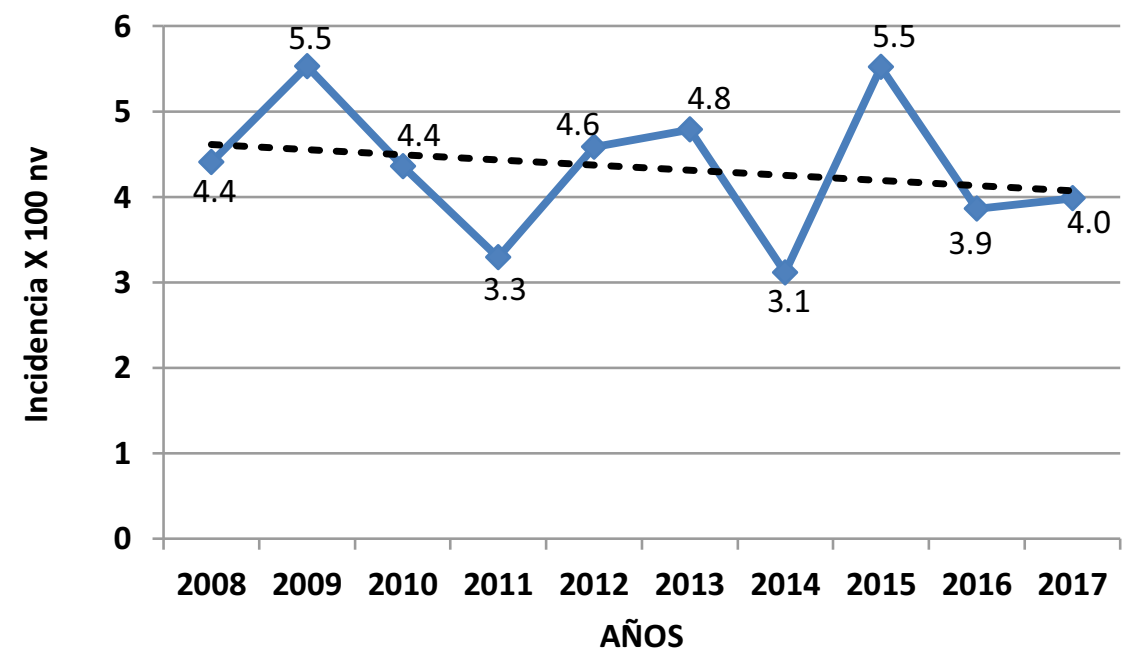


Tabla 2

Factores de riesgo socioeconómicos asociados con Apgar bajo al minuto

\begin{tabular}{|c|c|c|c|c|c|c|c|}
\hline \multirow{2}{*}{$\begin{array}{c}\text { Factores } \\
\text { socioeconómicos }\end{array}$} & \multicolumn{2}{|c|}{ Apgar < 7} & \multicolumn{2}{|c|}{ Apgar $7-10$} & \multirow{2}{*}{ OR } & \multirow{2}{*}{ IC } & \multirow{2}{*}{$p$} \\
\hline & $n$ & $\%$ & $n$ & $\%$ & & & \\
\hline \multicolumn{8}{|l|}{ Edad materna } \\
\hline 10 a 19 años & 214 & 14,1 & 5106 & 15,4 & 0,96 & $0,82-1,12$ & 0,58 \\
\hline 20 a 34 años & 1029 & 68,0 & 23543 & 71,2 & Ref. & & \\
\hline 35 años a más & 270 & 17,9 & 4441 & 13,4 & 1,39 & $1,21-1,60$ & 0,00 \\
\hline \multicolumn{8}{|l|}{$\begin{array}{l}\text { Grado de } \\
\text { instrucción }\end{array}$} \\
\hline Analfabeta & 2 & 0,1 & 71 & 0,2 & 0,62 & $0,11-2,58$ & 0,51 \\
\hline Primaria & 157 & 10,4 & 2883 & 8,7 & 1,20 & $1,01-1,40$ & 0,03 \\
\hline Secundaria & 1032 & 68,2 & 22830 & 69,0 & Ref. & & \\
\hline Superior & 322 & 21,3 & 7321 & 22,1 & 0,97 & $0,85-1,11$ & 0,67 \\
\hline \multicolumn{8}{|l|}{ Estado civil } \\
\hline Casada & 166 & 11,0 & 4107 & 12,4 & Ref. & & \\
\hline Conviviente & 1123 & 74,2 & 24627 & 74,4 & 1,13 & $0,95-1,34$ & 0,15 \\
\hline Soltera & 224 & 14,8 & 4371 & 13,2 & 1,27 & $1,03-1,56$ & 0,02 \\
\hline
\end{tabular}

Tabla 3

Factores de riesgo obstétricos asociados con Apgar bajo al minuto

\begin{tabular}{|c|c|c|c|c|c|c|c|}
\hline \multirow{2}{*}{ Factores obstétricos } & \multicolumn{2}{|c|}{ Apgar $<7$} & \multicolumn{2}{|c|}{ Apgar $7-10$} & \multirow{2}{*}{ OR } & \multirow{2}{*}{ IC } & \multirow{2}{*}{$p$} \\
\hline & $n$ & $\%$ & $n$ & $\%$ & & & \\
\hline \multicolumn{8}{|l|}{ Estado nutricional } \\
\hline Desnutrida $($ IMC < 18.5) & 21 & 1,4 & 407 & 1,2 & 1,12 & $0,70-1,78$ & 0,61 \\
\hline Normal (IMC 18.5-24.9) & 690 & 45,6 & 15002 & 45,3 & Ref. & & \\
\hline Sobrepeso (IMC 25-29.9) & 493 & 32,6 & 11801 & 35,7 & 0,91 & $0,81-1,02$ & 0,11 \\
\hline Obesidad (IMC $\geq 30$ ) & 309 & 20,4 & 5895 & 17,8 & 1,14 & $0,99-1,31$ & 0,06 \\
\hline \multicolumn{8}{|l|}{ Control prenatal } \\
\hline Sin CPN & 360 & 23,8 & 6661 & 20,1 & 1,03 & $0,91-1,18$ & 0,62 \\
\hline De 1 a 5 & 383 & 25,3 & 11721 & 35,4 & 0,62 & $0,55-0,71$ & 0,00 \\
\hline De 6 a más & 770 & 50,9 & 14723 & 44,5 & Ref. & & \\
\hline \multicolumn{8}{|l|}{ Paridad } \\
\hline Primípara & 705 & 46,6 & 14915 & 45,1 & 1,09 & $0,98-1,21$ & 0,12 \\
\hline Multípara (2-4) & 745 & 49,2 & 17150 & 51,8 & Ref. & & \\
\hline Gran Multípara (5 a más) & 63 & 4,2 & 1040 & 3,1 & 1,39 & $1,06-1,83$ & 0,01 \\
\hline \multicolumn{8}{|l|}{ Morbilidad materna } \\
\hline $\mathrm{Si}$ & 909 & 60,1 & 18111 & 54,7 & 1,25 & $1,12-1,39$ & 0,00 \\
\hline No & 604 & 39,9 & 14994 & 45,3 & Ref. & & \\
\hline \multicolumn{8}{|l|}{ Patologías maternas } \\
\hline Polihidramnios & 14 & 0,9 & 26 & 0,1 & 11,88 & $5,89-23,71$ & 0,00 \\
\hline Diabetes & 7 & 0,5 & 33 & 0,1 & 4,66 & $1,88-11,02$ & 0,00 \\
\hline Presentación fetal anómala & 103 & 6,8 & 685 & 2,1 & 3,46 & $2,78-4,30$ & 0,00 \\
\hline Hemorragias II mitad & 40 & 2,6 & 273 & 0,8 & 3,27 & $2,30-4,62$ & 0,00 \\
\hline RPM & 42 & 2,8 & 504 & 1,5 & 1,85 & $1,32-2,57$ & 0,00 \\
\hline Oligohidramnios & 21 & 1,4 & 279 & 0,8 & 1,66 & $1,03-2,63$ & 0,03 \\
\hline Enf. Hipertensiva del emb. & 54 & 3,6 & 804 & 2,4 & 1,49 & $1,11-1,98$ & 0,01 \\
\hline Anemia & 417 & 27,6 & 9414 & 28,4 & 0,96 & $0,85-1,08$ & 0,46 \\
\hline ITU & 392 & 25,9 & 8869 & 26,8 & 0,96 & $0,85-1,08$ & 0,45 \\
\hline
\end{tabular}




\section{Tabla 4}

Factores de riesgo del parto asociados con Apgar bajo al minuto

\begin{tabular}{|c|c|c|c|c|c|c|c|}
\hline \multirow{2}{*}{ Factores del parto } & \multicolumn{2}{|c|}{ Apgar $<7$} & \multicolumn{2}{|c|}{ Apgar 7-10 } & \multirow{2}{*}{ OR } & \multirow{2}{*}{ IC } & \multirow{2}{*}{$p$} \\
\hline & $n$ & $\%$ & $n$ & $\%$ & & & \\
\hline \multicolumn{8}{|l|}{ Terminación } \\
\hline Espontánea & 745 & 49,2 & 18339 & 55,4 & Ref. & & \\
\hline Cesárea & 768 & 50,8 & 14766 & 44,6 & 1,28 & $1,15-1,42$ & 0,00 \\
\hline \multicolumn{8}{|l|}{ Liquido amniótico } \\
\hline Claro & 1297 & 88,4 & 30966 & 93,5 & Ref. & & \\
\hline Verde claro & 131 & 8,9 & 1758 & 5,3 & 1,78 & $1,47-2,15$ & 0,00 \\
\hline Verde oscuro & 40 & 2,7 & 381 & 1,2 & 2,51 & $1,78-3,53$ & 0,00 \\
\hline \multicolumn{8}{|l|}{ Duración parto vaginal } \\
\hline Prolongado & 3 & 0,4 & 28 & 0,2 & 2,75 & $0,66-9,46$ & 0,08 \\
\hline Normal & 713 & 98,2 & 18274 & 99,6 & Ref. & & \\
\hline Precipitado & 10 & 1,4 & 39 & 0,2 & 6,57 & $3,06-13,72$ & 0,00 \\
\hline
\end{tabular}

Tabla 5

Factores de riesgo perinatales asociados con Apgar bajo al minuto

\begin{tabular}{|c|c|c|c|c|c|c|c|}
\hline \multirow{2}{*}{ Factores perinatales } & \multicolumn{2}{|c|}{ Apgar $<7$} & \multicolumn{2}{|c|}{ Apgar 7-10 } & \multirow{2}{*}{ OR } & \multirow{2}{*}{ IC } & \multirow{2}{*}{$\mathrm{p}$} \\
\hline & $n$ & $\%$ & $n$ & $\%$ & & & \\
\hline \multicolumn{8}{|l|}{ Sexo RN } \\
\hline Masculino & 903 & 59,7 & 16949 & 51,20 & 1,41 & $1,27-1,57$ & 0,00 \\
\hline Femenino & 610 & 40,3 & 16156 & 48,80 & & & \\
\hline \multicolumn{8}{|l|}{ Peso RN } \\
\hline $\begin{array}{l}\text { Bajo peso }(<2500 \text { g. }) \\
\text { Peso insuficiente }(2500-\end{array}$ & 385 & 25,5 & 1109 & 3,4 & 10,86 & $9,45-12,47$ & 0,00 \\
\hline 2999 g.) & 171 & 11,3 & 3169 & 9,6 & 1,69 & $1,42-2,01$ & 0,00 \\
\hline $\begin{array}{l}\text { Peso adecuado (3000- } \\
3999 \text { g.) }\end{array}$ & 763 & 50,4 & 23864 & 72,0 & Ref. & & \\
\hline Alto peso (4000 g. a más) & 194 & 12,8 & 4963 & 15,0 & 1,22 & $1,04-1,44$ & 0,01 \\
\hline \multicolumn{8}{|l|}{ Edad gestacional } \\
\hline Pretérmino (< 37 sem.) & 426 & 28,2 & 1383 & 4,2 & 9,01 & $7,94-10,22$ & 0,00 \\
\hline A término (37-41 sem.) & 1083 & 71,6 & 31677 & 95,7 & Ref. & & \\
\hline Postérmino (42 a más) & 4 & 0,2 & 45 & 0,1 & 2,60 & $0,79-7,55$ & 0,05 \\
\hline \multicolumn{8}{|l|}{ Patología perinatal } \\
\hline Malformaciones cong. & 108 & 7,1 & 344 & 1,0 & 7,32 & $5,82-9,20$ & 0,00 \\
\hline RCIU & 105 & 6,9 & 399 & 1,2 & 6,11 & $4,87-7,67$ & 0,00 \\
\hline Gemelaridad & 37 & 2,5 & 358 & 1,1 & 2,29 & $1,60-3,27$ & 0,00 \\
\hline
\end{tabular}

